\title{
Proposed Design on Driver Behavioral Analysis
}

\author{
Jessica Bhalerao ${ }^{1}$ \\ Student, Computer Dept. \\ Pimpri Chinchwad College of Engineering
}

Pune, India.

\author{
Vaishnavi Mugalikar ${ }^{4}$ \\ Student, Computer Dept. \\ Pimpri Chinchwad College of Engineering \\ Pune, India
}

\author{
Archana Kadam ${ }^{2}$ \\ Asst. Professor, Dept. of \\ Computer Engineering, Pimpri \\ Chinchwad College of Engineering, \\ Pune, India. \\ Amruta Shinde ${ }^{3}$, \\ Student, Computer Dept. \\ Pimpri Chinchwad College of Engineering \\ Pune, India.
}

\author{
Himanshu Bhan ${ }^{5}$ \\ Student, Computer Dept. \\ Pimpri Chinchwad College of Engineering \\ Pune, India
}

Dept. of Computer Engineering, Pimpri Chinchwad College of Engineering, Nigdi, Maharashtra, India

\begin{abstract}
Currently, in tune with economic growth in every country, the number of vehicles increase every year. At the same time, the number of non-experts drivers also increases rapidly, since most novice drivers are unskilled, unfamiliar with the vehicle conditions and no awareness of traffic rules and regulations, driver's personal factors have become the main reason of traffic accidents. Considering all the consequences that could eventuate after an accident, there are reasons to believe that a normal person does not drive with an intention to cause an accident but his/her behaviour on the road while driving surely reflect the consequences. In this paper, we propose a system architecture that aims to combine driver background data and driving data, driver drowsiness and distraction as well as various environmental factors affecting driver's behaviour in order to analyze and evaluate driving behaviours patterns. Whereas for predicting driver action we propose a system that predicts a safe or unsafe driver using prediction models based on the analysis carried. To demonstrate the idea, we utilize logistic regression to analyze drive background data, computer vision to analyze driver behaviour based on environmental factors, that is from off-line data samples collected during course of driving and SVM to analyze drowsiness. By analyzing the given factors, we utilize HMM for predicting driving behaviour. Although traditional system is bounded to single parameter, as results of comparison with traditional system, out system is effective for predicting driving behaviour accurately by the driver behaviour analysis and the driver behaviour.
\end{abstract}

Keywords : Driving behaviour, logistic regression, AHP-Entropy, Support Vector Machine (SVM), Hidden Markov Model (HMM), Viola-Jones.

\section{INTRODUCTION}

Modern vehicles are becoming more intelligent through the application of different types of vehicle active safety technologies. It can be envisioned in the future these new technologies will significantly reduce today's traffic problems. However, drivers' bad driving behavior currently is still a serious problem, when advanced intelligent cars (e.g., autonomous vehicles) are not able to fully replace human drivers. Understanding human's driving Behavior remains of great interests in the research community in the last decade, the number of improvements for driver safety has been more than ever yet a significant number of accidents still occur all over the world. Those accidents are mostly caused by human mistakes, For eg:- typing a text message, speaking someone on the phone, eating and drinking etc., while driving. In addition to these activities, drowsiness, sleepiness, distraction, driver background data and driving data, surrounding conditions could also result in critical and harsh accidents.

Traffic accidents can cause physical, financial and mental damages for those who involved in it. Drivers considering more than one parameter. Understanding and passengers can suffer from minor cuts and bruises to human's driving behaviour remains of great interests in broken limbs, whiplash, back and spinal injuries, the research community. In this paper, we have carried paralysis and even death. Vehicles in traffic accidents

out a detailed survey about

are damaged and may be in need of minor or costly repairs or may even be wreckage and no longer drivable.Statistical data given by British and American crash reports show us that avoiding driving during drowsiness and/or distraction is extremely important approach to prevent such serious accidents. Driver's background (driving experience, average driving distance per year, and number of violations of traffic rules, etc.) and driving habits (frequency of sudden acceleration/deceleration, overspeed, sharp turning, etc.)would both affect the cause accidents. Road surface condition, weather, traffic density may also be responsible for accidents.

In this paper, we aim to focus on the analysis of driverbehaviour and based on the analysis predict whether driver is a safe driver or not? And for analysis we consider more than one parameter. For analysis we consider a parameters like distraction and drowsiness, driver background data and driving data, surrounding conditions. The goal is to create a system that predict driver's behavior that whether driver is a safe driver or not. And will help to reduce number of accidents.

Our proposed model will be a hybrid system which can take more than one parameter such as driver background data and driving data, environmental factors, distraction and 
drowsiness at once. And based on this system will also predict whether the driver is safe driver or not. So that will help in reducing level of accidents occurring and also will save many lives. And also which can be further used to develop a system which will alarm a driver which will help to reduce number of accidents. We will be using methodologies like Support Vector Machine, Viola jones, Face Detection ,Logistic Regression, Computer Vision, HMM,AHP entropy and Entropy Weight.

\section{LITERATURE SURVEY :}

There have been a number of data collection, analysis and prediction efforts on driver's behavior prior to this research. Those efforts can be roughly divided into four groups i.e. drowsiness and distraction of driver, drivers background data and driving data analysis, drivers surrounding conditions, prediction based on drivers behaviour. The paper[5] determine whether the standard used to detect drowsiness can also be used to predict when a given drowsiness level will be reached. We explore whether adding data like driving time and participant information improves the accuracy of detection and prediction of drowsiness. Two significant models were developed using artificial neural networks, one to detect the degree of drowsiness every minute, and the second to predict every minute the time required to reach a particular drowsiness level (moderately drowsy). The model can detect the drowsiness level and can predict when a given drowsiness level will be reached.The different algorithms used here are k-nearest neighbors, decision trees, Bayesian classifiers Support Vector Machines artificial neural networks (ANN) ensemble methods like random forest etc. With these techniques, the model can extract information from noisy data, and can avoid over-fitting, making it generally more robust.Since in the context of driving we expected overfitting and noisy data, the present study uses machinelearning techniques based on artificial neural networks.Using these algorithm we have to predict when a given impaired state will bereached in addition to detecting this impaired state.

In this[3] paper, they have proposed a system architecture that aims to combine driver background data and driving data, in order to analyze and evaluate driving behaviors. For this, we used utilize logistic regression to analyze drive background data, apply the analytic hierarchy process and entropy weight technique to assess driving data, and output scores to indicate different levels of good/bad driving patterns. In this paper, we aim to adopt the big data concept to facilitate driving behavior analysis. Consider that a driver's background (driving experience, average driving distance per year, and number of violations of traffic rules, etc.) and driving habits (frequency of sudden acceleration/deceleration, over-speed, sharp turning, etc.) would both affect the possibility of having accidents. A system architecture is proposed to combine driver background data and driving data collected from onboard sensors, to generate a score that indicates whether the driver is prone to cause traffic accidents. The final score consists of driver background score and driving habits score.
Specifically, we apply logistic regression to connect drivers' background with the probability of accidents occurrence, so that a score can be provided on a driver's background data. The analytic hierarchy process (AHP) and

entropy weight technique is used to evaluate the impact of different driving habits from both subjective and objective perspectives. Finally, a radar plot is generated to illustrate which aspects contribute to the score. Potential applications of the system may include providing drivers with feedbacks to improve their driving behaviors, and offering references to personalized vehicle insurance, or active safety applications in IoV systems mean square error of 4.1 min.This Paper [1] considers a project to collects large driving data for use in a wide range of areas of vehiclerelated research centered on driving behavior. Unlike to the previous, the corpora collected here contain both human and vehicle sensor data, together with rich and continuous transcriptions.

Details relating to the data collection paradigm, such assensors, driver information, routes, and transcription protocols, are discussed, and studied a preliminary analysis of the data across the three data collection sites. In this paper authors adopted computer vision algorithm to group near crash events according to their driving risk. Face recognition is also done with the help of computer vision andalso with the help of the Bayesian network the frustration level is measured which affects drivers behavior.

This paper[6]has investigated the driving behavior differences at un-signalized intersections between expert and non expert drivers. By analyzing real- world data, significant differences were seen in pedal operations but not in steering operations. . The paper propose two prediction models in terms of traffic conditions and driver types: one is based on multiple linear regression analysis.In second Bayesian Network is used to predict driver decelerating intentions using a Bayesian Network. . The decelerating intentions correspond to pedal taps and the switch timing of the gas and brake pedals. In the experiments, participants drove a data collection vehicle on city roads that included many unsignalized intersections. The prediction models were evaluated by comparing the predicted driving behaviors with the actual behaviors of target driver. 
3. PROPOSED ARCHITECTURE:

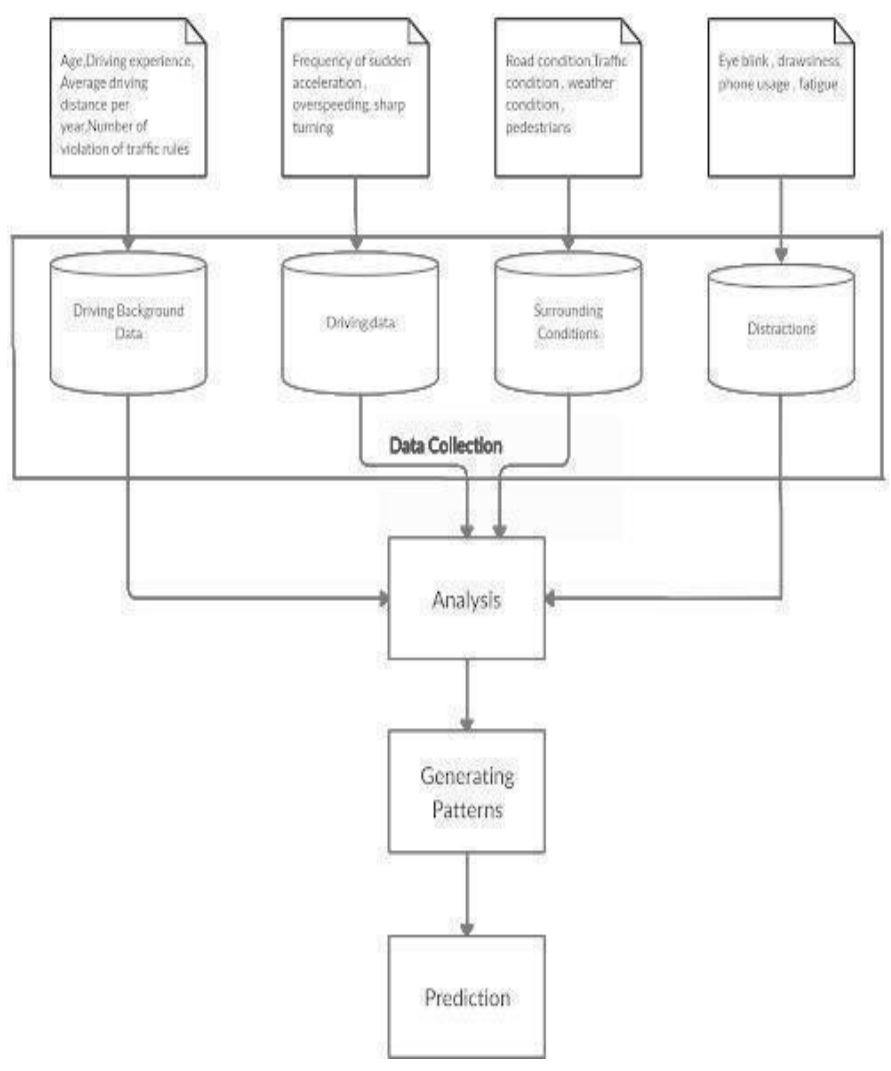

Fig.3.1 Proposed architecture diagram

\subsection{PROPOSED METHODOLOGY}

One of the most challenging problems faced today is the increase in traffic accidents and financial loss. Many techniques are proposed to overcome this problem but there is not a hybrid system made till even today that would consider all the parameters at once. We propose to use different techniques like Support Vector Machine(SVM), Viola-jones face Detection, LinearRegression, HMM,AHP-Entropy, Bayesian Network ,Computer Vision and K- clustering to achieve our hybrid System. In our hybrid system we are considering four parameters like driving background data, driving data surrounding conditions and distraction and based on these parameters we will perform analysis .Based on these analysis we will generate patterns .And based on those patterns we will predict whether driver is a safe driver or not.

\subsection{Viola-jones Face Detection with SVM:}

Our proposed solution uses Viola Jones for face detection, PCA with Multilevel Grid search method is used for facial data processing and feature extraction. Further SVM is applied on data for classification. Proposed model identifies faces more accurately with the accuracy of $85 \%$ compared to current traditional method. Experimental results are measured based on the sensitivity, specificity and precision using the lfw-deep funneled dataset.
Viola-Jones algorithm scan the sub window of the image and detect face images across the input images. And then fixed size detector runs through these images but first rescale the input image to different sizes.

\section{(HMM) Hidden-Markov-Model:}

HMM is a statistical model which is used to model time series data, in which current state of the system is hidden and follows the Markovian process. HMM is well suited for such scenario efficient learning, as the model learning takes place directly from the raw sequence data. Trained the model based on accidents data-set, as well as other driving scenarios. From Simulation results we concluded that the proposed system has a better performance.

\section{Computer Vision Algorithm:}

It is multidisciplinary field that could broadly be called a subfield of artificial intelligence and machine learning, which may involve the use of specialized methods and make use of general learning algorithms.

A simple example can be finding the edges in an image. Computer vision is one of the areas in Machine Learning where core concepts are already being integrated into major products that we use every day.

\section{Logistic regression:}

The logistic regression is a predictive analysis. categorical. For example in the prediction of email is spam or not. Logistic regression measures the relationship between the categorical dependent variable and one or more independent variables by estimating probabilities using a logistic function, which is the cumulative distribution function of logistic distribution. It treats the same set of problems as probit regression using similar techniques, with the latter using a cumulative normal distribution curve instead.

\section{SUMMARY}

Therefore different algorithms and techniques have been studied based on various parameters which include driver background data and driving data, distraction and drowsiness, environmental factors.

We can say that the existing system is in good tone with its respective aims and objectives, but with certain additions and implementations of Machine Learning algorithms, as discussed, it can be made more helpful and more accurate for the assistance of the professionals by considering more than one parameters and applying prediction model on it as discussed in proposed system.

To conclude we can say that, by removing limitation of existing system considering more than one parameter for analysis we have proposed a new system which will do analysis based on more than one parameter such as driver background data and driving data, distraction and drowsiness, environmental factors and also apply prediction model to check whether driver is a safe driver 


\section{REFERENCES}

[1] Kazuya Takeda, John H. L. Hansen, , Pınar Boyraz, Lucas Malta, Chiyomi Miyajima, and Hüseyin Abut, "International Large-Scale Vehicle Corpora for Research on Driver Behavior on the Road'IEEETransactionsonIntelligent

TransportationSystems,VOL.12,NO.4,DECEMBER.

[2] Abdalla Abdelrahman, Najah Abu-Ali, Hossam S. Hassanein "On the Effect of Traffic and Road Conditions on the Drivers' Behavior: A Statistical Analysis", IEEE, 2018.

[3] Jiayin Cen, Zhuowen Wang, Chao Wang, Fuqiang Liu, "A System Design for Driving Behavior Analysis and Assessment", School of Electronics and Information Engineering Tongji University Shanghai, China, 2016

[4] Apoorva Mishra, Dr. Preeti Bajaj “Driver's Behaviour Monitoring on Urban Roads of a Tier 2 City in India", International Conference on Emerging Trends in Engineering \& Technology,2015.

[4] Sinan Kaplan, Mehmet Amac Guvensan, Member, IEEE, Ali Gokhan Yavuz, and Yasin Karalurt, "Driver Behavior Analysis for Safe Driving: A Survey", IEEE Transactions on Intelligent Transportation Systems, 2015.

[5] Hideomi Amata, Chiyomi Miyajima, Takanori Nishino, Norihide Kitaoka, and Kazuya Takeda, "Prediction Model of Driving Behavior Based on Traffic Conditions and Driver Types", 2019.

[6] Movements Yoshifumi Kishi moto, and Koji Oguri, "A Modeling Method for Predicting Driving Behavior Concerning with Driver's Past", 2008.

[7] Satoshi Hyodoa, Tohio Yoshiib,Matstushita Satoshib,Shirayanagi Hirotoshib, "An analysis of the impact of driving time on the driver's behavior using probe car data.",2017. 